\title{
Coefficient of variation and its application to strength prediction of self-piercing riveted joints
}

\author{
Xiaocong He \\ Faculty of Mechanical and Electrical Engineering, Kunming University of Science and Technology, Kunming, 650093, P. \\ R. China. E-mail: hhxxcc@yahoo.co.uk, xiaocong_he@163.com. Tel: +86-871-5170917, +86-1588787075. \\ Fax: +86-871-5194243. \\ Accepted 28 September, 2011

\begin{abstract}
This study deals with an application of the method of the coefficient of variation in strength prediction of the self-piercing riveted joints. Defined as the ratio of the standard deviation to the mean, the coefficient of variation may be used in both the reliability-based design of self-piercing riveted joints and in the evaluation of existing products. In this study, the concept and definition of the coefficient of variation are stated. The procedure of the use of coefficient of variation for approximate calculations of tensile strength of the self-piercing riveted joints is presented and compared with the classical Taylor Expansion method. This is illustrated with a numerical example.
\end{abstract}

Key words: Coefficient of variation; self-piercing riveted joint; strength prediction; statistical parameter; approximate calculation.

\section{INTRODUCTION}

Due to the need to design lightweighted structures such as aircraft panels and vehicle body shells, and due to the increased use of lightweight sheet materials, some joining techniques have been developed for joining advanced lightweight materials that are dissimilar, coated, and hard to weld ( $\mathrm{He}$ et al., 2008, 2010 and 2011). Self-piercing riveting (SPR) has also been developed rapidly into a new branch of sheet materials joining techniques.

The SPR process is a cold forming operation used to fasten two or more sheets of material by driving a semitubular rivet through the top sheets piercing the bottom sheet and spreading the rivet skirt under the guidance of a suitable die. As the process relies on a mechanical interlock rather than fusion, it can be used for a wide range of advanced materials that are dissimilar, coated and hard to weld. Figure 1 shows the schematic representation of the SPR process. Lennon et al. (1999) have carried out shear tests on four types of mechanical connections; these are self-pierce riveting, press joining, pop riveting and self-tapping screws, with sheet thicknesses 1.0, 1.2, 1.6 and $2.0 \mathrm{~mm}$. The results show that self-pierce riveting produces a high-peak load, a high initial stiffness and high ductility compared to the other processes. Tang et al. (2002) proposed a method which consists of establishing baseline strength characteristics in the six degrees of freedom and modifying the baseline properties with coefficient factors that influence the performance of SPR processes. In the paper of Sun et al. (2005), the limit load based strength estimator was used to estimate the static strength of the SPR joints under a cross-tension loading condition. The rivet strength estimator was then used to optimize the rivet strength by comparing the measured rivet strength and failure mode with the predicted ones.

The strength of the SPR joints is affected by a number of factors including substrate thickness and mechanical properties, direction of applied load, and the geometric configuration of the SPR tools. Therefore, in the reliability-based SPR joints design, multivariate functions, which contain several random variables, may be met frequently. The statistical parameters of the multivariate functions may be found by using the functional relation of each random variable. However, as the functional relation is often very complicated, it is difficult to derive it using theoretical method (Riley et al., 1997). The complicated functional relationship may be transformed into simple form by using the concept of coefficient of variation (COV) (He and Oyadiji, 2001).

The concept of the COV simplifies greatly the calculation of statistical parameters and the results obtained are very close to those derived directly from the 

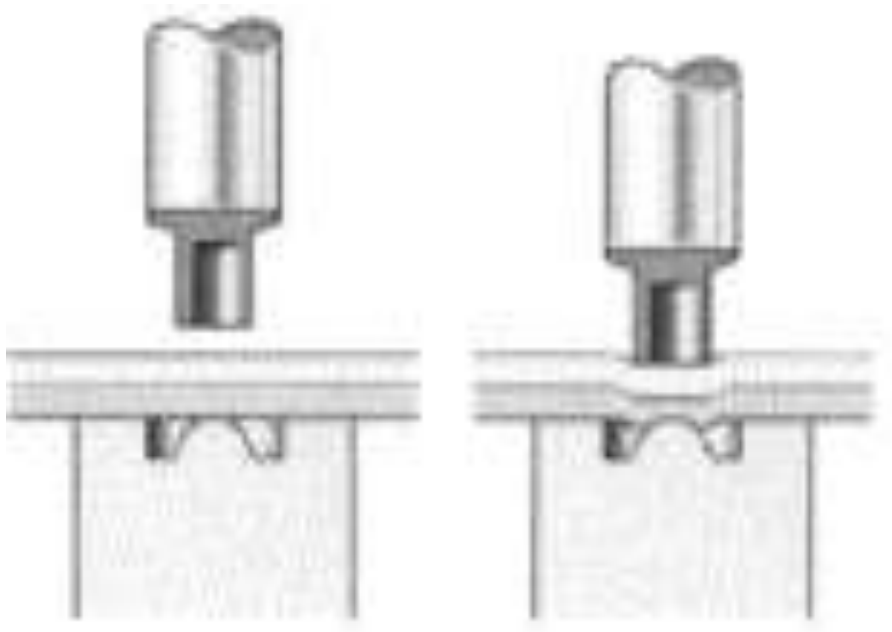

Figure 1. The principle of self-piercing riveting

original functional relation. In this paper, the concept and definition of the COV are stated. The procedure of the use of the COV for approximate calculations of SPR joint tensile strength is then presented and compared with the classical Taylor expansion method. By means of a numerical example, it is shown that the method of the COV can give an identical result to that of the Taylor expansion method.

\section{APPLICATION OF THE COV IN THE APPROXIMATE CALCULATION OF STATISTICAL PARAMETERS}

\section{Definition of the COV}

If $X$ is a random variable with probability density function $f(x)$, where $X$ is a specific value of $X$, then the mean value of the probability distribution of $X$ is:

$$
\mu=\int_{-\infty}^{\infty} x f(x) d x
$$

The variance is:

$$
\sigma^{2}=\int_{-\infty}^{\infty}(x-\mu)^{2} f(x) d x
$$

And it can be rewritten as:

$$
\sigma^{2}=\int_{-\infty}^{\infty} x^{2} f(x) d x-\mu^{2}
$$

The ratio of the standard deviation, which is the square root of the variance $\sigma^{2}$, to the mean value is the coefficient of variation, $C$, given by:

$$
C=\sigma / \mu
$$
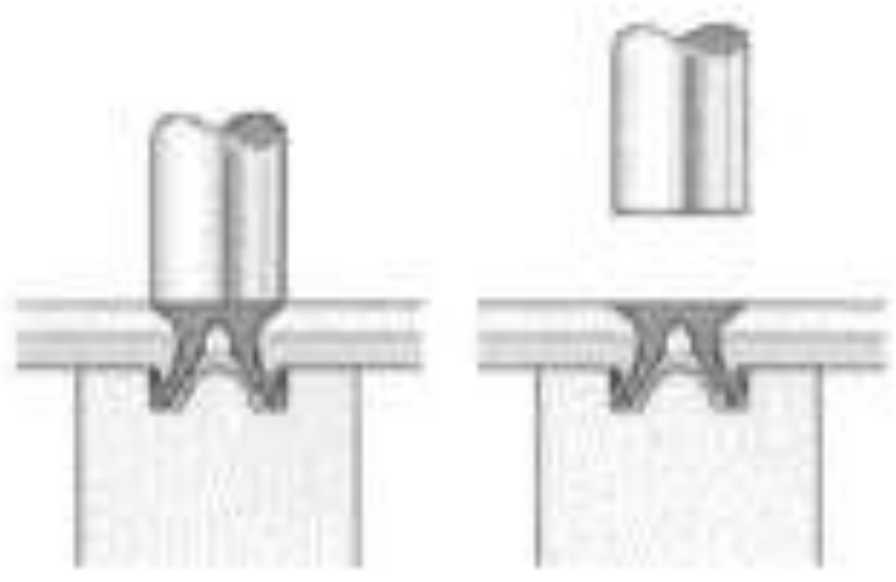

\section{Taylor expansion method}

This method is widely used in the approximate calculation. The random function is expanded in Taylor series. Then, the mean value and the standard deviation may be calculated approximately by using the first few terms of the series. Let the random function be $y=g(x)$. If the probability density function $f(x)$ is known, then from equations (1) and (2), the mean value and the variance of $y$ may be obtained by:

$\mu_{y}=\int_{-\infty}^{+\infty} g(x) f(x) d x$

$\sigma_{y}^{2}=\int_{-\infty}^{+\infty}\left[g(x)-\mu_{y}\right]^{2} f(x) d x$

If $f(x)$ is unknown, then $\mu_{\mathrm{y}}$ may be only obtained by using Taylor expansion method. By expanding $g(x)$ as a Taylor series centered on the mean value $\mu_{\mathrm{x}}$ of $x$ and only using the first two terms, we have:

$g(x) \approx g\left(\mu_{\mathrm{x}}\right)+g^{\prime}\left(\mu_{\mathrm{x}}\right)\left(x-\mu_{\mathrm{x}}\right)$

Substituting Equation (7) into Equation (5)

$\mu_{y} \approx \int_{-\infty}^{+\infty}\left[g\left(\mu_{\mathrm{x}}\right)+g^{\prime}\left(\mu_{\mathrm{x}}\right)\left(x-\mu_{\mathrm{x}}\right)\right] f(x) d x$

$=g\left(\mu_{x}\right)$

Then substituting Equations (7) and (8) into Equation (6)

$$
\begin{aligned}
& \sigma_{y}^{2} \approx \int_{-\infty}^{+\infty}\left[g\left(\mu_{\mathrm{x}}\right)+g^{\prime}\left(\mu_{\mathrm{x}}\right)\left(x-\mu_{\mathrm{x}}\right)-g\left(\mu_{\mathrm{x}}\right)\right]^{2} f(x) d x \\
& =\left[g^{\prime}\left(\mu_{\mathrm{x}}\right)\right]^{2} \sigma_{\mathrm{x}}^{2}
\end{aligned}
$$

For the multivariate function consisting of many independent random variables, by using a similar method, we may deduce: 


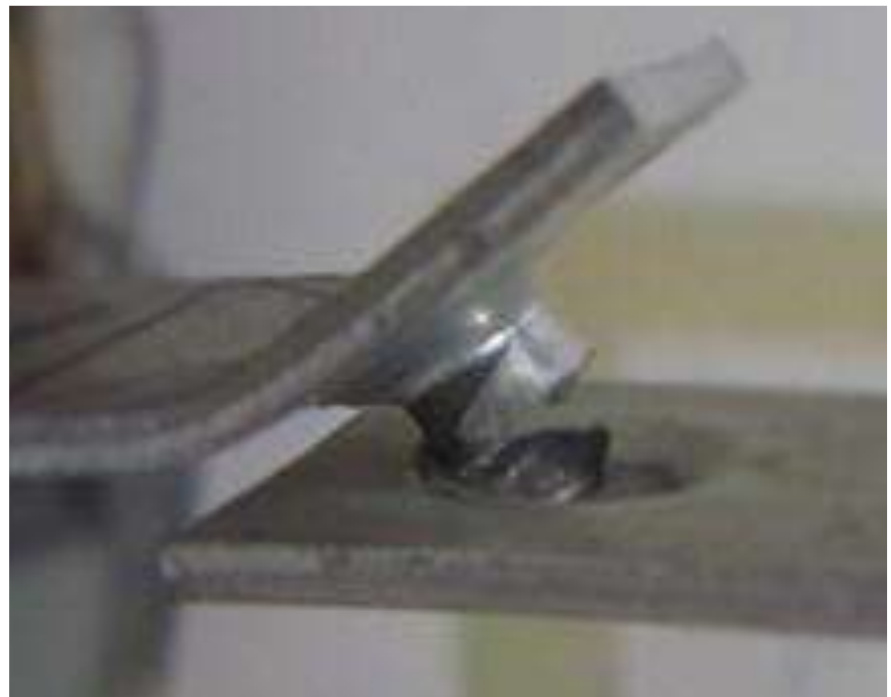

Figure 2. Typical failure modes of SPR joint: rivet tail pull-out.

$$
\begin{aligned}
& \mu_{y} \approx g\left(\mu_{\mathrm{x}_{1}}, \mu_{\mathrm{x}_{2}}, \ldots, \mu_{\mathrm{x}_{n}}\right) \\
& \sigma_{y}^{2} \approx \sum_{\mathrm{i}=1}^{\mathrm{n}}\left(\frac{\partial g}{\partial x_{\mathrm{i}}}\right)_{0}^{2} \sigma_{\mathrm{x}_{\mathrm{i}}}^{2}
\end{aligned}
$$

Where $\mu_{\mathrm{x}_{\mathrm{i}}}, \sigma_{\mathrm{x}_{\mathrm{i}}}^{2}$ are the mean value and the variance of; $\mathrm{x}_{\mathrm{i}}$, $\left(\frac{\partial g}{\partial x_{\mathrm{i}}}\right)_{0}$ is the value of $\frac{\partial \mathrm{g}}{\partial \mathrm{x}_{\mathrm{i}}}$ at the point

$$
\left(\mathrm{x}_{1}, \mathrm{x}_{2}, \ldots, \mathrm{x}_{\mathrm{n}}\right)=\left(\mu_{\mathrm{x}_{1}}, \mu_{\mathrm{x}_{2}}, \ldots, \mu_{\mathrm{x}_{\mathrm{n}}}\right)
$$

Thus it can be seen that when evaluating the standard deviation of multivariate function, the use of partial derivatives is inevitable. Therefore the calculation is very complicated.

\section{The method of the COV}

For the common monomial multivariate function in the reliabilitybased mechanical design, the calculation may be simplified greatly by using the method of the COV. For the multivariate function consisting of many independent random variables:

$$
y=a x_{1}^{\mathrm{m}_{1}} \cdot x_{2}^{\mathrm{m}_{2}} \cdot \ldots \cdot x_{\mathrm{n}}^{\mathrm{m}_{\mathrm{n}}}=a \prod_{\mathrm{i}=1}^{\mathrm{n}} x_{\mathrm{i}}^{\mathrm{m}_{\mathrm{i}}}
$$

Where $a$ and $m_{i}$ are positive or negative constants. By using the $\mathrm{COV}$ in the Taylor expansion method which is discussed before, the

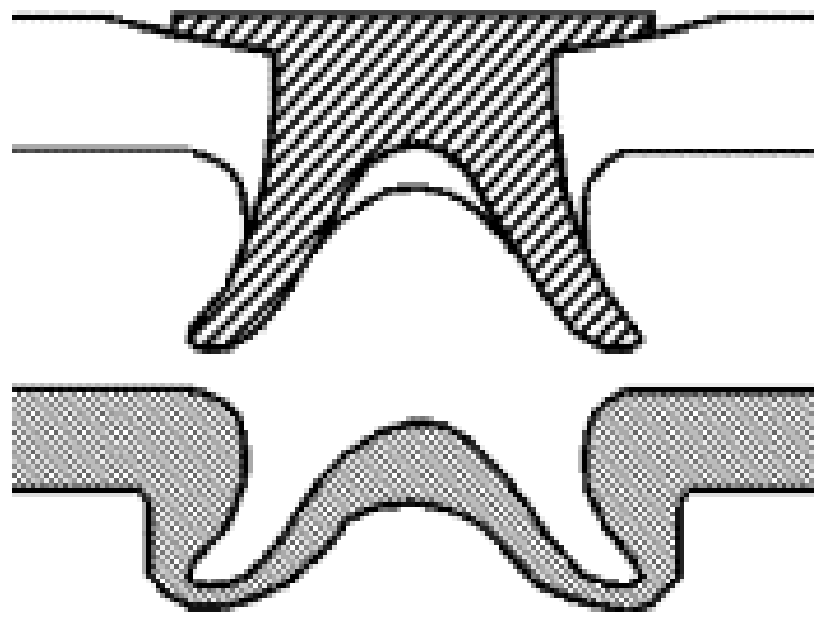

determination of the standard deviation may be simplified greatly. They are:

$$
\begin{aligned}
& \mu_{y} \approx a \prod_{\mathrm{i}=1}^{\mathrm{n}} \mu_{\mathrm{x}_{\mathrm{i}}}^{\mathrm{m}_{\mathrm{i}}} \\
& C_{y}^{2} \approx \sum_{\mathrm{i}=1}^{\mathrm{n}} m_{\mathrm{i}}^{2} C_{\mathrm{x}_{\mathrm{i}}}^{2} \\
& \sigma_{y}=\mu_{y} C_{y}
\end{aligned}
$$

Thus, the complication of finding partial derivatives is avoidable by using the coefficient of variation.

\section{Application of the COV in strength prediction of SPR joints}

Typically, there are two failure modes of SPR joints, namely rivet tail pull-out and rivet head pull-out, as shown in Figures 2 and 3 . In the case of the rivet tail pull-out, the rivet tail is going out from the bottom substrate dragging material from the bottom substrate in contact with the rivet shank. In the case of the rivet head pull-out, the rivet head goes out from the top substrate dragging the material under the rivet head. The complex riveted joint geometry and its three dimensional nature, combine to increase the difficulty of obtaining an overall system of governing equations for predicting the strength of SPR joints. Porcaro et al. (2006) proposed the following relationship, based on the tensile resistance formulae present in the Eurocode 9 (2004), for both the rivet tail pull-out and rivet head pull-out resistance:

$$
F=\alpha_{p} f_{u} \sqrt{d t^{3}}
$$

Where $f_{u}$ is the ultimate tensile strength of the substrate, $d$ is the nominal diameter of the un-deformed rivet shank and $t$ is the thickness of the substrate. For the case of the rivet tail pull-out, $f_{u}$ 


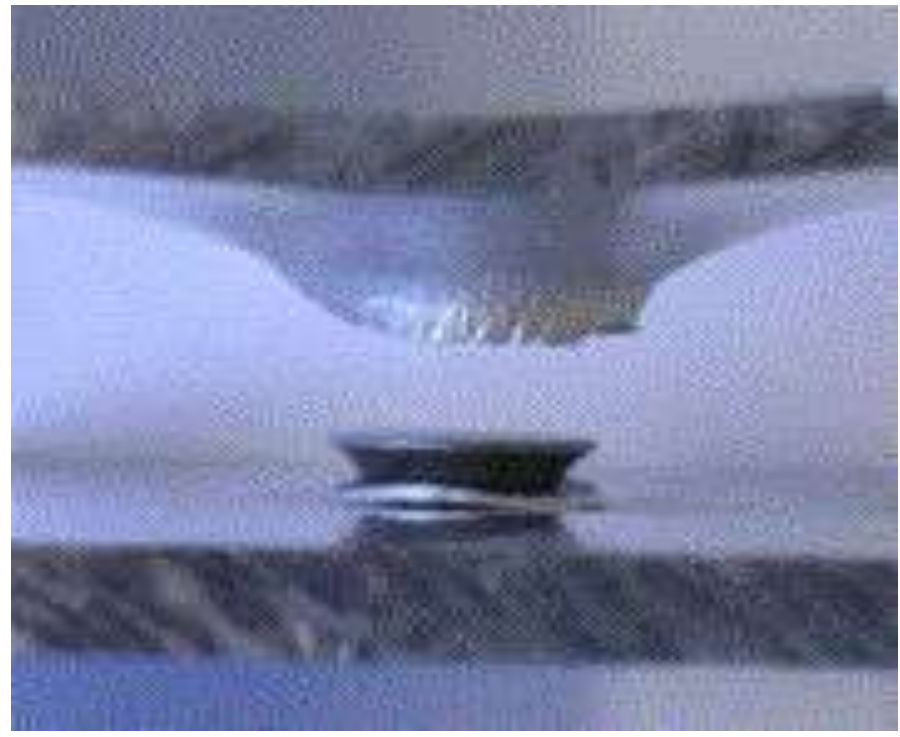

Figure 3. Typical failure modes of SPR joint: rivet head pull-out.

and $t$ of the bottom substrate should be used in Equation 15. While for the rivet head pull-out resistance, average values of $f_{u}$ and $t$ of the top and bottom substrates should be used. The results from a large number of tests show that the average value of the coefficient $\alpha_{p}=2.35$ can be used for both the rivet tail pull-out condition and rivet head pull-out condition (Porcaro et al., 2006). Generally the rivets are made of much stronger material than the substrates and rivet shank deformation rarely occurs in actual static overloading tests. The nominal rivet diameter $5 \mathrm{~mm}$ is used in this study. Thus the tensile strength of the SPR joints can be given by:

$$
F=5.25 f_{u} t^{1.5}
$$

In the case of single lap-jointed SPR joints, the parameters $f_{u}$ and $t$ are independent random variables, and $F$ is the dependent random variable. The mean values and variances of the independent random variables are as follows: $\mu_{f_{u}}$ and $\sigma_{f_{u}}^{2}$ for the ultimate tensile strength $f_{u}, \mu_{\mathrm{t}}$ and $\sigma_{t}^{2}$ for the substrate thickness $t$. Let the means and the standard deviations of the independent variables have the following numerical values: $\mu_{f_{u}}=220 \mathrm{MPa}, \sigma_{f_{u}}=10$ MPa, $\mu_{\mathrm{t}}=2 \mathrm{~mm}, \sigma_{t}=0.15 \mathrm{~mm}$. The problem now is to find the mean value $\mu_{F}$ and the standard deviation $\sigma_{F}$ of the strength of the clinched joints $F$.

\section{Using the Taylor expansion method}

From Equations (10) and (16):

$$
\mu_{F} \approx 5.25 \mu_{f_{u}} \mu_{t}^{1.5}
$$

Substituting the numerical values of the means gives:

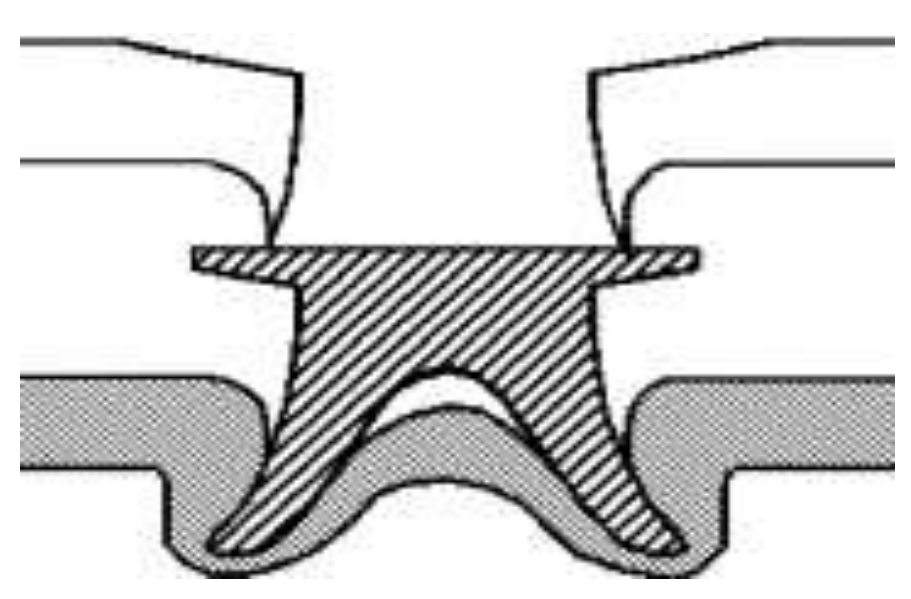

$\mu_{F}=5.25 \times 220 \times 2^{1.5}=3266.83 \mathrm{MPa}$

From Equation (11):

$$
\begin{aligned}
& \sigma_{F}^{2} \approx\left[\frac{\partial}{\partial f_{u}}\left(5.25 f_{u} t^{1.5}\right)\right]^{2} \sigma_{f_{u}}^{2}+\left[\frac{\partial}{\partial t}\left(5.25 f_{u} t^{1.5}\right)\right]^{2} \sigma_{t}^{2} \\
& =\left(5.25 \times \mu_{t}^{1.5}\right)^{2} \sigma_{f_{u}}^{2}+\left(5.25 \times \mu_{f_{u}} \times 1.5 \times \mu_{t}^{0.5}\right)^{2} \sigma_{t}^{2}
\end{aligned}
$$

Substituting the numerical data in equation (18) gives:

$\sigma_{F}^{2}=\left(5.25 \times 2^{1.5}\right)^{2} \times 10^{2}+\left(5.25 \times 220 \times 1.5 \times 2^{0.5}\right)^{2} \times 0.15^{2}$ $=157120.03(\mathrm{MPa})^{2}$

Which gives $\sigma_{F}=396.38 \mathrm{MPa}$. It is obvious that the calculation is very complicated.

\section{Using the method of the coefficient of variation}

From Equation (12) and (16):

$\mu_{F} \approx 5.25 \mu_{f_{u}} \mu_{t}^{1.5}$

Which gives $\mu_{F}=3266.83 \mathrm{MPa}$ as earlier mentioned.

From equation (13):

$C_{F}^{2} \approx 1^{2} \times C_{f_{u}}^{2}+1.5^{2} \times C_{t}^{2}$

For the given values: 
$C_{f_{u}}=\sigma_{f_{u}} / \mu_{f_{u}}=0.04545$,

$C_{t}=\mu_{t} / \mu_{t}=0.075$,

Thus;

$C_{F}^{2}=1^{2} \times 0.04545^{2}+1.5^{2} \times 0.075^{2}=0.01472225$

Then, $C_{F}=0.1213$

The standard deviation is given by:

$\sigma_{F}=\mu_{F} C_{F}$

Substituting:

$\sigma_{F}=3266.83 \times 0.1213=396.27 \mathrm{MPa}$

Which is almost the same as the value obtained by the Taylor expansion method.

\section{DISCUSSION}

It is seen that the COV method is much simpler than that of using the Taylor expansion in SPR tensile strength prediction. From the dimension of each random variable in the coefficient of variation, the level of importance of each variable can also be appraised and compared with the level of importance of other variables. Moreover, the COV method will be very useful in the future SPR fatigue strength prediction. In general, the fatigue strength of SPR joints follows Weibull distributions. For example, a two-parameter Weibull distribution is used here. The probability density function of a two-parameter Weibull distribution is given by:

$$
f(x)=\frac{\alpha}{\beta}\left(\frac{x}{\beta}\right)^{\alpha-1} \exp \left[-\left(\frac{x}{\beta}\right)^{\alpha}\right]
$$

Where $\alpha$ is the shape parameter and $\beta$ the scale parameter. From equations (1), (2) and (21), the mean value and the variance of the two-parameter Weibull distribution can be deduced as:

$$
\begin{aligned}
& \mu=\beta \Gamma\left(1+\frac{1}{\alpha}\right) \\
& \sigma^{2}=\beta^{2}\left\{\Gamma\left(1+\frac{2}{\alpha}\right)-\left[\Gamma\left(1+\frac{1}{\alpha}\right)\right]^{2}\right\}
\end{aligned}
$$

Then, the coefficient of variation, $C$, given by:

$$
C=\left\{\frac{\Gamma(1+(2 / \alpha))}{[\Gamma(1+(1 / \alpha))]^{2}}-1\right\}^{1 / 2}
$$

Where $\Gamma($.$) is the gamma function. It can be seen from$ equation (24) that $C$ is only a function of $\alpha$ and independent from $\beta$. In the range 1-50, equation (24) can be written approximately as:

$C \approx \alpha^{-0.93}$

From equation (24), the approximate relation between the coefficient of variation $C$ and the statistical parameters can be found for a certain range of precision. Therefore, the coefficient of variation can be used to study the characteristics of the probability distribution. On the contrary, the coefficient of variation can be evaluated by using these approximate relations if the statistical parameters are known.

Fatigue testing is usually used to assess the fatigue strength of SPR joints by destructive testing of joints. Obviously destructive testing is not acceptable for large number of specimens as it is expensive and timeconsuming. When the number of specimens tested in each category is small, the usual method of determination of Weibull parameters is difficult. However, following the COV method the values of the Weibull shape and scale parameters can be evaluated by equations (22) and (25). Therefore, the COV method is useful in finding the statistical parameters of multivariate functions in the SPR fatigue strength prediction.

\section{CONCLUSION}

Based on the original definition of the coefficient of variation, this study discusses the quality of utility and simplicity of it. It is possible to study the characteristic of the common probability distribution in the reliability-based mechanical design with it, even to use it directly as the strength index of SPR joints. Additionally, the COV may be used to compare the discreteness of the experimental data under different conditions and parameters (Jeong, 2006). In the future research work, the COV will be applied to experimental data inorder for it to be obtained from SPR joints. This will enable a prediction of the fatigue life of SPR joints.

\section{ACKNOWLEDGEMENT}

This study is partially supported by National Science Foundation of China (Grant No. 50965009)

\section{REFERENCES}

Eurocode 9 (2004). CEN, prEN1999-1-4, Design of aluminium structures, Part 1-4: Supplementary rules for cold-formed sheeting (Stage 34), May 2004.

$\mathrm{He} X$ (2010). Recent Development in Finite Element Analysis of Clinched Joints. Int. J. Adv. Manuf. Technol., 48: 607-612.

$\mathrm{He}$ X (2011). A Review of Finite Element Analysis of Adhesively Bonded 
He X, Oyadiji SO (2001). Application of Coefficient of Variation in Reliability-Based Mechanical Design and manufacture. J. Mater. Process. Technol., 119: 374-378.

He X, Pearson I, Young K (2008). Self-pierce riveting for sheet materials: State of the art. J. Mater. Process. Technol., 199: 27-36.

Jeong $J(2006)$. Effect of specimen shape on the behavior of brittle materials using probabilistic and deterministic methods. J. Eur. Ceramic. Soc., 26: 3621-3629.

Joints. Int. J. Adhes. Adhes, 31: 248-264.

Lennon R, Pedreschi R, Sinha BP (1999). Comparative study of some mechanical connections in cold formed steel. Constr. Build. Mater., 13(3): 109-116.

Porcaro R, Hanssen AG, Langseth M, Aalberg A (2006). An experimental investigation on the behaviour of self-piercing riveted connections in aluminium alloy AA6060. Int. J. Crash., 11(5): 397-417. Riley KF, Hoson MP, Bence SJ (1997). Mathematical Methods for Physics and Engineering. Cambridge University Press. Cambridge.

Sun X, Khaleel MA (2005). Performance optimization of self-piercing rivets through analytical rivet strength estimation. J. Manuf. Process., 7(1): 83-93.

Tang D, Barthelemy B, Yuan H (2002). Self-pierced rivet (SPR) modeling in aluminum structure crash analysis. In: Proceedings of the ASME International Mechanical Engineering Congress and Exposition IMECE 2002, November 17-22, New Orleans, LA, pp. 207-222. 